\title{
Global prevalence of Trichomonas vaginalis among female sex workers: a systematic review and meta-analysis
}

\author{
Monirsadat Mirzadeh ${ }^{1} \cdot$ Meysam Olfatifar $^{2} \cdot$ Aida Vafae Eslahi $^{3} \cdot$ Amir Abdoli $^{4} \cdot$ Elham Houshmand $^{5}$. \\ Hamidreza Majidiani ${ }^{6}$. Morteza Ghanbari Johkool ${ }^{1}$. Setareh Askari ${ }^{7}$. Sima Hashemipour ${ }^{1} \cdot$ Milad Badri $^{8}$
}

Received: 23 March 2021 / Accepted: 8 June 2021 / Published online: 25 June 2021

(C) The Author(s), under exclusive licence to Springer-Verlag GmbH Germany, part of Springer Nature 2021

\begin{abstract}
Trichomoniasis is a sexually transmitted infection (STI), caused by the protozoan parasite, Trichomonas vaginalis. Female sex workers are intensely affected by the infection, since they have frequent direct physical contact. The current systematic review and meta-analysis represents the global prevalence of $T$. vaginalis in female sex workers. Five databases (Science Direct, Scopus, PubMed, Web of Science, and Google Scholar) were explored for literatures that published from July 1985 to June 2020. Totally, 85 studies (54,515 participants) from 46 countries met the inclusion criteria. The global pooled prevalence of $T$. vaginalis was $16 \%$ (95\% CI 13-19\%). The estimated pooled prevalence based on methods including wet mount, culture, and molecular techniques was $15 \%$ (95\% CI 12-19\%), $16 \%$ (95\% CI 10-24\%), and $22 \%$ (95\% CI 13-32\%), respectively. Moreover, the infection was most prevalent at the mean age of 30-36 (20\%, 95\% CI 11-30\%). Regarding the World Health Organization (WHO) regions, the highest pooled prevalence was estimated to be in the African region (23\%, 95\% CI 7-46\%). In addition, we indicated that countries with low-income level have the highest pooled prevalence (23\%, 95\% CI 14-34\%). Our results revealed that the worldwide prevalence of $T$. vaginalis was significant in female sex workers. Therefore, considering a precise strategy such as a health education program with regard to safe intercourse is needed to increase knowledge and prevent $T$. vaginalis infection in sex workers.
\end{abstract}

Keywords Trichomoniasis $\cdot$ Worldwide epidemiology $\cdot$ Female sex workers $\cdot$ Sexually transmitted diseases

Monirsadat Mirzadeh and Aida Vafae Eslahi contributed equally to this work.

Section Editor: Kevin S.W. Tan

Sima Hashemipour

hashemipour.sima@yahoo.com

Milad Badri

Badri22.milad@gmail.com

1 Metabolic Diseases Research Center, Research Institute For Prevention Of Non-Communicable Diseases, Qazvin University Of Medical Sciences, Qazvin, Iran

2 Gastroenterology and Liver Diseases Research Center, Research Institute for Gastroenterology and Liver Diseases, Shahid Beheshti University of Medical Sciences, Tehran, Iran

3 Clinical Research Development Unit, Velayat Hospital, Qazvin University Of Medical Sciences, Qazvin, Iran
4 Zoonoses Research Center, Jahrom University of Medical Sciences, Jahrom, Iran

5 Department of Parasitology, Faculty of veterinary medicine, Rasht Branch, Islamic Azad University, Rasht, Gilan, Iran

6 Zoonotic Diseases Research Center, Ilam University of Medical Sciences, Ilam, Iran

7 Department of Medical Parasitology \& Mycology, School of Public Health, Tehran University of Medical Science, Tehran, Iran

8 Medical Microbiology Research Center, Qazvin University of Medical Sciences, Qazvin, Iran 